\title{
Identification Potency of Clinical Isolates in Aspergillus Species Using MALDI-TOF MS
}

\author{
Norihito Tarumoto $^{1,2 *}$, Jun Sakai ${ }^{1,2}$, Keisuke Sujino ${ }^{1,2}$, Takuya Maeda ${ }^{2,3}$, Mayu Ohta ${ }^{1,2}$, Toshiyuki Yamaguchi ${ }^{1,2}$, Takashi \\ Murakami $^{2,3}$, Hideaki Ohno ${ }^{2,4}$ and Shigefumi Maesaki ${ }^{1,2}$ \\ ${ }^{1 *}$ Department of Infectious Disease and Infection Control, Saitama Medical University, Saitama, Japan \\ ${ }^{2}$ Center for Clinical Infectious Diseases and Research, Saitama Medical University, Saitama, Japan \\ ${ }^{3}$ Department of Microbiology, Saitama Medical University, Saitama, Japan \\ ${ }^{4}$ Department of Infectious Disease and Infection Control, Saitama Medical Center, Saitama Medical University, Japan
}

${ }^{*}$ Corresponding author: Norihito Tarumoto, MD, PhD., Department of Infectious Diseases and Infection Control, Saitama Medical University, 38 Morohongo, Moroyama-machi, Iruma-gun, Saitama 350-0495, Japan; Phone: +81-49-276-2032, Fax: +81-49-276-1188

Received: June 19, 2017; Accepted: June 26, 2017; Published: June 26, 2017;

\begin{abstract}
It is important to make accurate identification of genus Aspergillus including cryptic species, because they could exhibit drug resistance to multiple antifungal agents. Recent advances in molecular diagnosis extended to clinical mycology, and matrix-assisted laser desorption/ionization time-offlight mass spectrometry (MALDI-TOF MS) has been applied as an attractive methodology. Herein we evaluated the ability of MALDI-TOF MS for the identification of genus Aspergillus. A total of 42 strains of Aspergillus were genetically identified by the sequence analysis of PCR amplicons and simultaneously followed by MALDI-TOF MS. The concordance rate for Aspergillus using MALDI-TOF MS was $90.5 \%$ in comparison with sequencing of PCR amplicons. Thirty eight registered strains in the MALDI Biotyper standard library showed the perfect concordance rate (100\%). Notablely, azolesresistant Aspergillus lentulus and Aspergillus felis, which were not registered in the MALDI Biotyper standard library, failed to be identified as the correct species. Moreover, these species with low score values in the MALDI-TOF MS analysis potentially resisted to a variety of antifungal agents. These results suggest that additional drug susceptibility testing should be further considered in poor yields with MALDI-TOF MS analysis.
\end{abstract}

Keywords: matrix-assisted laser desorption/ionization time-of-flight mass spectrometry; Aspergillus felis; Aspergillus lentulus; Aspergillus tubingensis; cryptic species

\section{Introduction}

The genus Aspergillus is one of the ubiquitous fungi that exhibit wide clinical spectrums of diseases in humans. It is a causative pathogen from an allergic response to subacute invasive life-threatening diseases, depending on the host immune conditions. Chronic aspergillosis including aspergilloma is usually found in patients with previously formed lung cavities and/or with mild immunocompromised status. Especially the lives of immunocompromised patients (receiving organ transplantation and anti-cancer chemotherapy) could be threatened by invasive aspergillosis (IA). Additionally, as IA often results in a fatal outcome, the early diagnosis and immediate treatment for IA with optimal chemotherapy lead to improvement of patient's prognosis $[1,2]$.

Antifungal agents often exhibit variable activities against fungal organisms. For example, a few strains of genus Aspergillus could acquire drug resistance to multiple antifungal drug classes in accordance with failure of the antifugal treatment [3]. In addition, it has also been reported that azole-resistant cases of Aspergillus fumigatus have been increasing in number with several mutations in azole target genes [4]. Furthermore, cryptic species of A. fumigatus tend to resist azole antifungals, leading to the reduced treatment efficacy [5]. Therefore, in an effort to survey aspergillosis appropriately and to treat the patients with optimal antifungals, it is quite important to make accurate identification of genus Aspergillus including cryptic species.

Recently, matrix-assisted laser desorption ionization-time-offlight mass spectrometry (MALDI-TOF MS) (Bruker Daltonics, $\mathrm{BD}$, Bremen, Germany) has also been applied to the identification of fungal pathogens in clinical settings [6]. This methodology is based on applying a laser to the mixed crystals of the clinical specimens and matrix, before implementing acceleration with an electric field [7]. The flight time is then measured to determine the molecular weight of specimens and the pathogens could be identified with the reference of the previously registered molecular patterns in the library. While the use of MALDI-TOF MS has a great potential as one of the reliable methods to detect pathogens, the information on its detection sensitivity and specificity remains to be limited. In this study, we demonstrate the possibility and the current limitation for MALDITOF MS-mediated identification of genus Aspergillus.

\section{Materials and methods}

Our current study was conducted with clinical strains of genus Aspergillus that were previously isolated and identified in our hospital 
between April 2014 and August 2016. The isolated strains, cultured at $37^{\circ} \mathrm{C}$ on Sabouraud dextrose broth for 2 days, were suspended in Sabouraud dextrose broth with $20 \%$ glycerol and then were stored at $-80^{\circ} \mathrm{C}$ until the use.

For genetic identification of genus Aspergillus, stored samples were re-harvested on Potato Dextrose Agar (PDA) for 3 days, and each obtained colony was washed with $3 \mathrm{~mL}$ of phosphate buffered saline (PBS). DNA was then extracted from the pellet using the DNeasy plant mini kit (Qiagen, Hilden, Germany) according to the manufacturer's instructions. The extracted DNA was used for the conventional sequence analysis of the following PCR products for the identification of genus Aspergillus. Segments of the internal transcribed spacer (ITS) and D1/D2 region were amplified using the primers ITS1 and NL4 [8], a segment of the beta-tubulin gene was amplified using the primers bT2a and bT2b [9] and a segment of the calmodulin gene was additionally amplified using the primers cmd5 and cmd6 [10]. The sequencing analysis of these PCR products with blastn (v2.5.0) algorism against database nucleotide collection $(\mathrm{nr} / \mathrm{nt})$ confirmed the species of Aspergillus. Additionally, to distinguish Aspergillus oryzae and Aspergillus flavus, a segment of the transcriptional regulator gene of the aflatoxin biosynthesis genes was additionally amplified and performed sequencing analysis using the primers aflR F2 and aflR R2 [11].

Analysis with the MALDI-TOF MS system was applied to above obtained each colony according to the manufacturer's instructions with a MALDI Biotyper 3.1 RTC software and MALDI Biotyper 4.0 standard library (Bruker Daltonics, Bremen, Germany). Any score value (SV) of less than 1.7 was deemed insufficient for identification.

Drug susceptibilities were confirmed in accordance with the modified M38-A2 method, which is the standard protocol of the Clinical and Laboratory Standards Institute (CLSI) [12], with the lowest concentration of antibiotics without visible growth on the microplate of the Yeast-like Fungus DP Eiken Kit (Eiken Chemical, Tokyo, Japan): micafungin (MCFG), caspofungin (CPFG), amphotericin B (AMPH-B), itraconazole (ITCZ) and voriconazole (VRCZ) [12,13]. The breakpoint of each antifungal agent for Aspergillus was determined using the EUCAST Antifungal Agents Breakpoint tables for interpretation of minimum inhibitory concentrations (MICs) v. 8.0 [14]. For the epidemiological cut-off value (ECV) have been prescribed in this study as follows; $0.5 \mu \mathrm{g} / \mathrm{mL}$ was considered for CPFG [15]. The quality control was ensured by concurrent testing with the strain of A. fumigatus ATCC MYA-3626, which is a recommended strain of CLSI [12].

\section{Results}

A total of 42 strains of Aspergillus were genetically identified ( $\geq 99 \%$ identity) based on the sequence analysis of PCR amplicons from clinical specimens (Table 1). They consisted of 27 A. fumigatus, 7 Aspergillus niger, 3 Aspergillus terreus, 2 Aspergillus lentulus, and an each of A. oryzae, Aspergillus tubingensis and Aspergillus felis. To verify whether the results identified by MALDI-TOF MS could match the results by sequencing analysis, all isolates were simultaneously analysed by MALDI-TOF MS. As showin in Table 1, 38 out of 42 isolates ( 90.5 $\%)$ were mached as same spiceses of the genus Aspergillus. However, 2 specimens from cryptic species of $A$. fumigatus (A. lentulus and $A$. felis) showed very low score values (below 1.7) in MALDI-TOF MS analysis. Of note, these were not registered in the MALDI Biotyper standard library, and were unable to be correctly identified as its species. The score values for $A$. tubingensis, one of cryptic species of A. niger, were also below 1.772 and it was identified incorrectly as $A$. niger.

Table 1. Sequence analysis of Aspergillus isolates and application with MALDI-TOF MS

\begin{tabular}{|l|l|c|c|}
\hline \multirow{3}{*}{ Section } & \multicolumn{1}{|c|}{ Species } & $\begin{array}{c}\text { No. of isolates } \\
\text { confirmed with } \\
\text { PCR }\end{array}$ & $\begin{array}{c}\text { No. of matched } \\
\text { isolates } \\
\text { with TOF MS (\%) }\end{array}$ \\
\hline \multirow{3}{*}{ Fumigati } & Aspergillus fumigatus & 27 & $27(100)$ \\
\cline { 2 - 4 } & Aspergillus lentulus & 2 & $0(0)$ \\
\cline { 2 - 4 } & Aspergillus felis & 1 & $0(0)$ \\
\hline \multirow{2}{*}{ Nigeri } & Aspergillus niger & 7 & $7(100)$ \\
\cline { 2 - 4 } & Aspergillus tubingensis & 1 & $0(0)$ \\
\hline \multirow{2}{*}{ Terrei } & Aspergillus terreus & 3 & $3(100)$ \\
\hline \multirow{2}{*}{ Flavi } & Aspergillus oryzae & 1 & $38(100)$ \\
\hline \multirow{2}{*}{} & total & 42 & \\
\hline
\end{tabular}

We further addressed those drug susceptibilities in isolates that was incorrectly identified using MALDI-TOF MS. Results of the drug susceptibilities for Aspergillus section Fumigati were indicated in Table 2. For CPFG (ECV: $0.5 \mu \mathrm{g} / \mathrm{mL}$ ), the obtained MIC values of cryptic species for A. fumigatus ranged to above $4 \mu \mathrm{g} / \mathrm{mL}$ (A. lentulus) and $2 \mu \mathrm{g} / \mathrm{mL}$ (A. felis), whereas no resistance to CPFG was noted in A. fumigatus. For AMPH-B, 2 specimens of $A$. lentulus were resistant. For ITCZ, all specimens were ranged lower than breakpoint MIC. However, for VRCZ, the obtained MIC values even in two isolates of A. fumigatus ranged over the breakpoint. Furthermore, in each isolate of $A$. lentulus and $A$. felis, the obtained MIC value was 4 or $8 \mu \mathrm{g} / \mathrm{mL}$ [13-17].

\section{Discussion}

Recently, application of MALDI-TOF MS allowed accurate identification for pathogens at species levels and it is possible to discriminate them at strain levels including relatively genus Aspergillus 6,16,17) as well as common fungi (e.g., Candida spp. and Cryptococcus spp.) [18-20]. In this study, we demonstrated that the identification for Aspergillus with MALDI-TOF MS could obtain a high concordance rate $(90.5 \%)$, compared with the genetic identifications using PCR. However, some strains belonging to cryptic species remain not to be discriminated in this protocol. As the reason of this limitation in cryptic Aspergillus species, we raised that taxonomic references of those species were absent in the MALDI Biotyper standard library. Since cryptic Aspergillus species have emerged in clinical settings worldwide and they have high possibility to be resistant to azole and/ or polyene antifungal agents, more Aspergillus spp. including cryptic species should be resistered into the library of MALDI-TOF MS ${ }^{5)}$.

Aspergillus felis showed the lowest score values in our anlaysis, and others also failed the identification ${ }^{21)}$. A. lentulus and A. tubingensis were also unable to be identified and $A$. tubingensis was incorrectly identified as A. niger. Notablely, A. lentulus and A. felis showed the high MIC value for VRCZ (Table 2), and these results appeared to be consistent with the findings of previous studies [21,22]. Thus, we should consider further to perform drug resistance testing as 
confirmation for its susceptibilities, when the obtained score value (SV) is less than 1.7 or the pathogen identification failed based on the results of MALDI-TOF MS.

In conclusion, reconfiguration of database of MALDI-TOF MS in house is still required. As rare Aspergillus spp. including cryptic species have potencies of various antifungal resistance, additional drug susceptibility testing should be considered in poor yields with MALDI-TOF MS analysis.

\section{Acknowledgments: None}

\section{Conflict of Interest: None}

Table 2 Antifungal susceptibility for Aspergillus section Fumigati

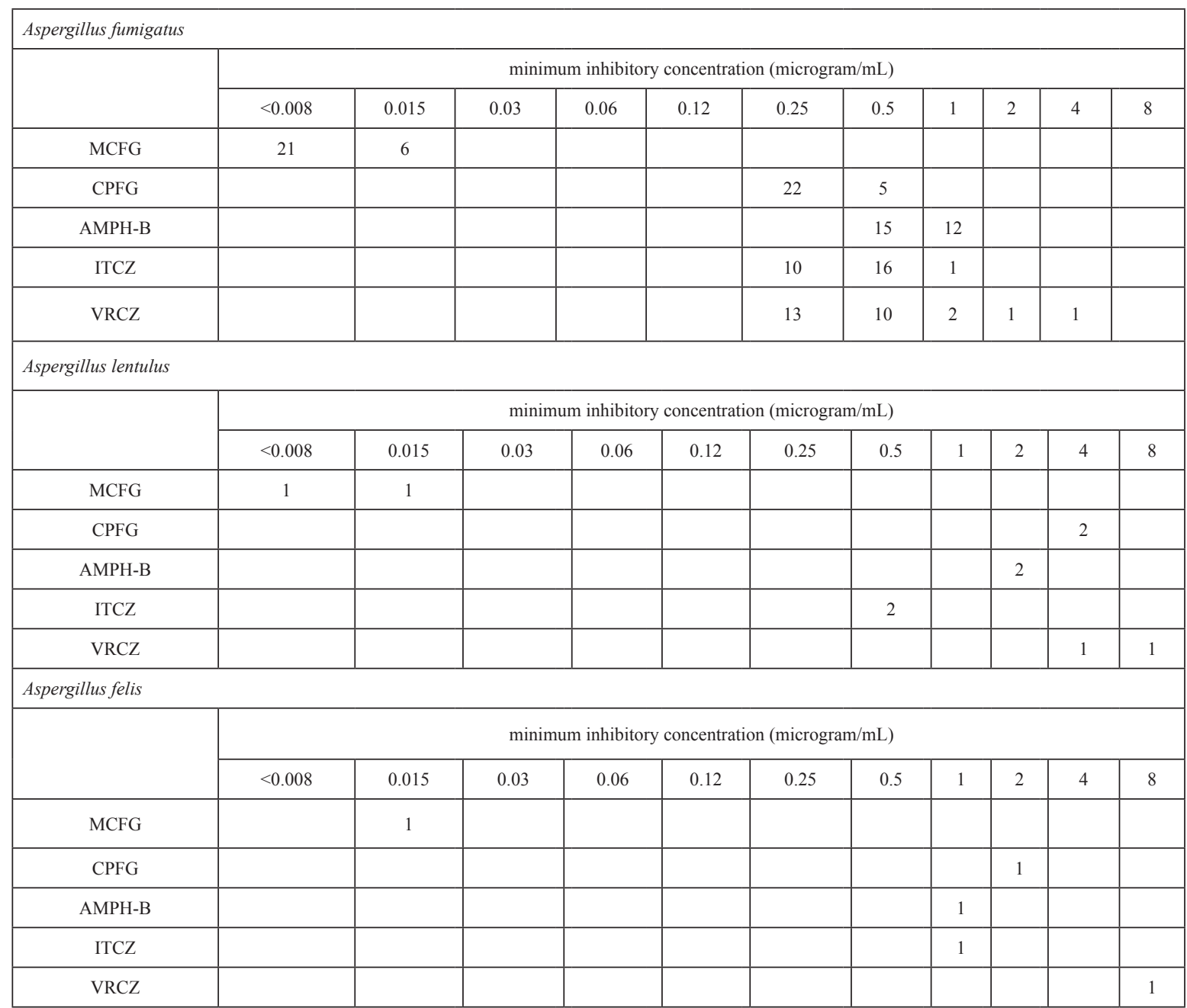

Abbreviations: MCFG, micafungin; CPFG, caspofungin; AMPH-B, amphotericin-B; ITCZ, itraconazole; VRCZ, voriconazole.

\section{References}

1. Lin SJ, Schranz J, Teutsch SM (2001) Aspergillosis case-fatality rate: systematic review of the literature. Clin Infect Dis 32: 358-366. [crossref]

2. Herbrecht R, Denning DW, Patterson TF, Bennett JE, Greene RE, Oestmann JW, et al. (2002) Voriconazole versus amphotericin B for primary therapy of invasive aspergillosis. N Engl J Med 347: 408-415.

3. Arendrup MC (2014) Update on antifungal resistance in Aspergillus and Candida. Clin Microbiol Infect 20 Suppl 6: 42-48. [crossref]

4. Snelders E, Karawajczyk A, Schaftenaar G, Verweij PE, Melchers WJ (2010) Azole resistance profile of amino acid changes in Aspergillus fumigatus CYP51A based on protein homology modeling. Antimicrob Agents Chemother 54: 2425-2430.

5. Alastruey-Izquierdo A, Alcazar-Fuoli L, Cuenca-Estrella M (2014) Antifungal susceptibility profile of cryptic species of Aspergillus. Mycopathologia 178: 427433.

6. Alanio A, Beretti JL, Dauphin B, Mellado E, Quesne G, Lacroix C, et al. (2011) Matrix-assisted laser desorption ionization time-of-flight mass spectrometry for fast and accurate identification of clinically relevant Aspergillus species. Clin Microbiol Infect 17: 750-755.
7. Tanaka K, Waki, H, Ido Y, Akita S, Yoshida Y, Yoshida T (1988) Protein and polymer analysis up to $\mathrm{m} / \mathrm{z} 100000$ by laser ionization time-of flight mass spectrometry. Rapid Communication in Mass Spectromeroty 2: 151-153.

8. O'Donnell K, Sutton DA, Fothergill A, McCarthy D, Rinaldi MG, Brandt ME, et al. (2008) Molecular phylogenetic diversity, multilocus haplotype nomenclature, and in vitro antifungal resistance within the Fusarium solani species complex. J Clin Microbiol 46: 2477-2490.

9. Glass NL, Donaldson GC (1995) Development of primer sets designed for use with the PCR to amplify conserved genes from filamentous ascomycetes. Appl Environ Microbiol 61: 1323-1330. [crossref]

10. Hong SB, Go SJ, Shin HD, Frisvad JC, Samson RA (2005) Polyphasic taxonomy of Aspergillus fumigatus and related species. Mycologia 97: 1316-1329. [crossref]

11. Tominaga M, Lee YH, Hayashi R, Suzuki Y, Yamada O, Sakamoto K, et al. (2006) Molecular analysis of an inactive aflatoxin biosynthesis gene cluster in Aspergillus oryzae RIB strains. Appl Environ Microbiol 72: 484-490

12. Clinical and Laboratory Standards Institute (2008) Reference method for broth dilution antifungal susceptibility testing for filamentous fungi; approved standard. Document M38-A2. Wayne, PA: Clinical and Laboratory Standards Institute. 
13. Toyotome T, Fujiwara T, Kida H, Matsumoto M, Wada T, Komatsu R (2016) Azole susceptibility in clinical and environmental isolates of Aspergillus fumigatus from eastern Hokkaido, Japan. J Infect Chemother 22: 648-650.

14. European Committee on Antimicrobial Susceptibility Testing (2015) Breakpoint tables for interpretation of MICs Version.

15. Espinel-Ingroff A, Fothergill A, Fuller J, Johnson E, Pelaez T, Turnidge J (2011) Wild-type MIC distributions and epidemiological cutoff values for caspofungin and Aspergillus spp. for the CLSI broth microdilution method (M38-A2 document). Antimicrob Agents Chemother 55: 2855-2859.

16. Cassagne C, Ranque S, Normand AC, Fourquet P, Thiebault S, Planard C, et al. (2011) Mould routine identification in the clinical laboratory by matrix-assisted laser desorption ionization time-of-flight mass spectrometry. PLoS One 6: e28425.

17. Lau AF, Drake SK, Calhoun LB, Henderson CM, Zelazny AM (2013) Development of a clinically comprehensive database and a simple procedure for identification of molds from solid media by matrix-assisted laser desorption ionization-time of flight mass spectrometry. J Clin Microbiol 51: 828-834.
18. Marklein G, Josten M, Klanke U, Muller E, Horre R, Maier T, et al. (2009) Matrixassisted laser desorption ionization-time of flight mass spectrometry for fast and reliable identification of clinical yeast isolates. J Clin Microbiol 47: 2912-2917.

19. Rosenvinge FS, Dzajic E, Knudsen E, Malig S, Andersen LB, et al. (2013) Performance of matrix-assisted laser desorption-time of flight mass spectrometry for identification of clinical yeast isolates. Mycoses 56: 229-235. [crossref]

20. Tarumoto N, Sakai J, Kodana M, Kawamura T, Ohno H, Maesaki S (2016) Identification of Disseminated Cryptococcosis Using MALDI-TOF MS and Clinical Evaluation. Med Mycol J 57: E41-46.

21. Barrs VR, van Doorn TM, Houbraken J, Kidd SE, Martin P, et al. (2013) Aspergillus felis sp. nov., an emerging agent of invasive aspergillosis in humans, cats, and dogs. PLoS One 8: e64871. [crossref]

22. Tamiya H, Ochiai E, Kikuchi K, Yahiro M, Toyotome T, Watanabe A, et al. (2015) Secondary metabolite profiles and antifungal drug susceptibility of Aspergillus fumigatus and closely related species, Aspergillus lentulus, Aspergillus udagawae, and Aspergillus viridinutans. J Infect Chemother 21: 385-39.

\section{Citation:}

Norihito Tarumoto, Jun Sakai, Keisuke Sujino, Takuya Maeda, Mayu Ohta, Toshiyuki Yamaguchi, Takashi Murakami, Hideaki Ohno and Shigefumi Maesaki (2017) Identification Potency of Clinical Isolates in Aspergillus Species Using MALDI-TOF MS. Internal Med Res Open J Volume 2(2): 1-4 